Babí Lladós, J.; Inglés Yuba, E.; Cumellas Ruiz, L.; Farías Torbidoni, E.I.; Seguí Urbaneja, J. y Labrador Roca, V. (2018). El perfil de los corredores y su propensión al accidente deportivo / Runner's Profile and Propensity to Sports Injury. Revista Internacional de Medicina y Ciencias de la $\begin{array}{llllllll}\text { Actividad Física y el Deporte vol. } 18 & \text { (72) pp. 737-752 }\end{array}$ Http://cdeporte.rediris.es/revista/revista72/artperfil964.htm DOI: http://doi.org/10.15366/rimcafd2018.72.009

\title{
ORIGINAL
}

\section{EL PERFIL DE LOS CORREDORES Y SU PROPENSIÓN AL ACCIDENTE DEPORTIVO}

\section{RUNNER'S PROFILE AND PROPENSITY TO SPORTS ACCIDENT}

Babí Lladós, J.'; Inglés Yuba, E. ${ }^{2}$; Cumellas Ruiz, L.. ${ }^{3}$ Farías Torbidoni, E.I.4; Seguí Urbaneja, J. ${ }^{4}$ Labrador Roca, V. ${ }^{2}$

${ }^{1}$ Personal Investigador del INEFC Barcelona (España) jbabi@gencat.cat

2 Doctores por la Universitat de Barcelona, profesor del INEFC Barcelona (España) eduard.ingles@gencat.cat, vlabrador@gencat.cat

${ }_{3}^{3}$ Profesor de la Universitat Ramón Llull - Blanquerna (España) lluiscr@blanquerna.url.edu

4 Doctores por la Universitat de Lleida, profesora del INEFC Lleida (España) efarias@inefc.es, jsegui@inefc.es

\section{FINANCIACIÓN Y AGRADECIMIENTOS}

Este trabajo forma parte del Proyecto de Investigación, con código 2014 PINEF 00006 y ha sido realizado con el apoyo del programa de becas predoctorales del Instituto Nacional de Educación Física de Cataluña (PINEFC-2015). Agradecemos el apoyo dado por el INEFC en la realización de este estudio, puesto que, sin su cobijo, no se hubiera podido llevar a cabo con las mismas condiciones.

Código UNESCO / UNESCO code: 6199 Otras especialidades psicológicas: Psicología del deporte/ Others: Sport Psychology

Clasificación Consejo de Europa / Council of Europe classification: 15 Psicología del deporte/ Sport Psychology

Recibido 25 de noviembre de 2016 Received November 25, 2016 Aceptado 3 de junio 2017 Accepted June 3, 2017

\section{RESUMEN}

Se evalúa las relaciones entre el perfil sociodemográfico, la accidentabilidad y la propensión al accidente de los participantes en tres eventos deportivos: Zurich Marató de Barcelona, Cros de Muntanya Can Caralleu, y Marató Borredà-Xtrail. Una adaptación del cuestionario de propensión al accidente deportivo (PAD-22) de Latorre y Pantoja (2013) fue administrado a un total de 237 corredores. Los principales resultados muestran que: los corredores tienden a ser mayoritariamente varones, de entre 30 y 46 años, asalariados, con estudios postobligatorios, con 
experiencia previa en eventos de larga distancia, entrenan una media de 4 veces y un total de 7 horas a la semana; y los corredores de la maratón por asfalto tienen una sobreestimación de la Competencia Percibida y grados de Competitividad mayores a los corredores por montaña.

PALABRAS CLAVE: carreras por montaña, propensión al accidente deportivo, prevención, riesgo de lesión, perfil de corredores.

\section{ABSTRACT}

This study evaluates relations between sociodemographic profile, accident rate and accident's propensity of three sport events participants: Zurich Marató de Barcelona, Cros de Muntanya Can Caralleu \& Marató Borredà-Xtrail. The used method was an adaptation of the sports accident prone scale (PAD-22) from Latorre y Pantoja (2013), to 237 runners. The main results show that: runners tend to be mostly men, aged of 30-46 years, are salaried, have post-compulsory studies, have some experience in long distance events, train a mean of 4 times and more than 7 hours per week; and marathon asphalt runners have a overestimation of Perceived Competence and elevated degrees of Competitiveness, more than trail runners.

KEY WORDS: trail races, accident propensity, prevention, risk of injury, runner's profile.

\section{INTRODUCCIÓN}

En los últimos años se ha producido un gran incremento de la práctica deportiva en entornos naturales (Clawson y Knetsch, 2013; González y Mundina, 2014). Este aumento ha sido generado por la creciente tendencia social a practicar deporte en espacios abiertos, como las montañas, los ríos, el mar, etc. -el $15,1 \%$ de la población española deportista, y el $24,1 \%$ de la población catalana practicante afirma realizar actividades físicas de aventura en el medio natural (García Ferrando y Llopis, 2011).

Las montañas han pasado de ser un espacio restringido a personas especialistas, muy bien preparadas físicamente, con un gran conocimiento y respeto por sus peligros y condiciones imprevisibles, a convertirse en un espacio deportivo sin límites, abierto a cualquiera que se atreva (Inglés, 2013).

El incremento global de la práctica del running (Llopis y Vilanova, 2015), ha provocado que la situación mencionada se ha visto todavía más potenciada por el aumento en la participación en las denominadas carreras por montaña o trail races. Así, por ejemplo, en el año 2008 se celebraron 38 carreras por montaña en Cataluña y en el año 2015 llegaron hasta 446; esto representa un aumento de más del 1000\% en 7 años (Runedia, 2016) Este crecimiento ha generado, en consecuencia, un aumento de los accidentes en montaña sufridos por corredores de nivel y experiencia diversos 0 , incluso, sin ninguna experiencia o preparación. En esta línea, se observa cómo ha aumentado el número de rescates realizados en Cataluña por el Grup de Recolzament d'Actuacions Especials (GRAE) de los 
Bomberos catalanes; se realizaron 387 rescates en la montaña en el año 2010, pasando a ser casi el doble, 755 rescates, en el 2014 (Generalitat de Catalunya, 2015). El presente estudio, ante esta alarmante situación, se plantea tratar de identificar los corredores por montaña que presentan una mayor propensión al accidente deportivo, y por lo tanto, al riesgo de sufrir una lesión, analizando la influencia del tipo de carrera y de la distancia en la que participan, teniendo en cuenta, también, variables sociodemográficas. En relación al sexo, por ejemplo, Byrnes, Miller, \& Schafer (1999) sugieren que las tendencias a la toma de riesgos de los hombres son superiores a la de las mujeres, siendo esta diferencia mayor cuando se trata de habilidades físicas.

De este modo, el objetivo principal de esta investigación es el de relacionar la propensión al accidente deportivo de los corredores analizados con sus características sociodemográficas y, sobre todo, centrando nuestra atención sobre las posibles diferencias acaecidas entre los corredores de asfalto o de montaña, así como entre una mayor o menor distancia recorrida.

\section{PERSPECTIVA TEÓRICA}

Hay varios estudios que explican los perfiles de los corredores en distintas modalidades. Hoffman y Fogard (2012), por ejemplo, dibujan el perfil mayoritario de los corredores de ultramaratones por montaña: hombres con un nivel alto de estudios, de mediana edad, casados, que raramente pierden días de trabajo a causa de enfermedades o lesiones, y que suelen utilizar vitaminas $y / 0$ suplementos, manteniendo una apropiada masa corporal ajustada a su edad. Por otro lado, en carreras populares sobre asfalto (Llopis \& Llopis, 2012; Llopis \& Vilanova, 2015) se define a los corredores como: jóvenes de entre 18 y 34 años, estudios universitarios, clase social alta o media-alta; en raids de aventura (Baena, 2008) el perfil mayoritario es de: varones de entre 26 y 30 años, solteros, asalariados o docentes en Educación Física, con estudios universitarios, pertenecientes a un club, y que realizan un entrenamiento diario y compiten a nivel nacional. No obstante, no hay estudios que relacionen el perfil de los corredores con la propensión al accidente deportivo.

Con el fin de contextualizar el estudio de la propensión al accidente deportivo, es importante entender que, por un lado, son múltiples los factores causantes de la lesión deportiva (Bahr y Krosshaug, 2005; Hanson, 1992; Heil, 1993; Meeuwisse, Tyreman, Hagel y Emery, 2007; Palmi, 2001), Andersen y Williams (1988) proponen un modelo predictivo de la lesión deportiva que diferencia tres grandes áreas causantes: la personalidad, el historial de factores estresantes y los recursos para afrontar el riesgo de lesión. Nuestro estudio se centra en el análisis de los factores psicológicos. Otros trabajos muestran los factores psicológicos de respuesta a la lesión deportiva, cuyos factores se asemejan a los observados en los estudios de predicción: cognitivos, emocionales y conductuales (Wiese-Bjornstal, Smith, Shaffer, \& Morrey, 1998).

Por otro lado, el concepto de lesión deportiva, desde la perspectiva psicológica, está definido por los siguientes componentes: disfunción o invalidez funcional producida durante la práctica deportiva, intervención del equipo médico-sanitario, y afectación en una parte o todo el entrenamiento y/o competición (baja deportiva) 
(Palmi, 2014), no obstante, los autores no utilizan la misma definición, en función de ser más o menos exigentes en el cumplimiento de los anteriores componentes. Por ello, y tal como apunta Palmi (2014), se delimita la definición de lesión deportiva que se utiliza para el presente trabajo, en el que entendemos la lesión deportiva como una disfunción o invalidez funcional producida durante la práctica deportiva sin tener en cuenta la intervención o no de médicos-sanitarios, ni el tiempo de afectación o baja deportiva.

Son abundantes los estudios que tratan de identificar distintos constructos psicológicos que definen a los deportistas. De la Vega, Rivera y Ruiz (2011) comparan los niveles de personalidad resistente de los participantes en la Ultra Trail Aneto, de 78 km, y La Melonera, una carrera popular de $10 \mathrm{~km}$; sin llegar a encontrar diferencias significativas entre sus participantes, y sin llegar a determinar que fuera un factor de influencia sobre el rendimiento en las carreras de ultrafondo. Hashimoto, Hagura, Kuriyama y Nishiyamai (2006) sugieren que los corredores de ultramaratones japoneses tienden a ser más introvertidos, toman decisiones, en mayor medida, basándose en sus sentimientos, y tienen más cuidado del medio ambiente que el resto de población japonesa.

En la línea de los trabajos realizados por Latorre y Pantoja (2013), se han definido 5 dimensiones psicológicas que determinan la posibilidad de sufrir un accidente deportivo:

1. Búsqueda de sensaciones (BS): es el rasgo de personalidad definido por la búsqueda de experiencias y sentimientos, que sean variadas, nuevas, complejas e intensas, y con disposición a tomar riesgos físicos, sociales, legales y financieros para satisfacer esas experiencias (Zuckerman, 1979, citado en Zuckerman 2009)(Zuckerman, 2009). Son muchos los autores que han realizado estudios sobre la BS mediante el instrumento Sensation Seeking Scale (SSS), elaborado por Zuckerman, Eysenck y Eysenck (1978). Gomà-i-Freixanet, Martha y Muro (2012) recopilan los estudios que han utilizado esta herramienta para su aplicación en diversos deportes, con el fin de saber cuál es el rasgo de personalidad de los deportistas y su relación con el nivel de riesgo físico del deporte en cuestión. Así, podemos observar como, por ejemplo, los alpinistas son considerados deportistas de riesgo elevado en comparación con los maratonianos, que son definidos como deportistas de bajo riesgo (Jack y Ronan, 1998). Por otro lado, Hughes, Case, Stuempfle y Evans (2003) definen a los participantes en ultramaratones como de riesgo intermedio al compararlos con estudiantes de universidad. Siguiendo la línea de estos estudios, Osborn, Blanton y Schwebel (2009) concluyeron que los deportistas con elevadas puntuaciones en BS pueden ser más propensos a tomar riesgos y a exponerse a situaciones potencialmente peligrosas con más frecuencia que los deportistas con bajas puntuaciones en BS; por lo que concluyen que a mayor propensión a la toma de riesgos, mayor será la posibilidad de sufrir un accidente deportivo.

2. Asunción de riesgos (AR). Horvath y Zuckerman (1993) sugieren que las experiencias pasadas exitosas con la AR pueden llevar a una reducción del riesgo percibido, aumentando potencialmente la posibilidad de que un deportista corra 
riesgos en el deporte. De este modo, podemos concebir la AR como el rasgo que define la capacidad consciente del sujeto para realizar situaciones arriesgadas.

3. Competencia percibida (CP). La autoeficacia y la competencia percibida pueden influir en el riesgo percibido y en la asunción de riesgos. En concreto, Bandura (1997) sugiere que los atletas que presentan una alta autoeficacia son más propensos a intentar habilidades difíciles, por lo tanto, son más propensos a tomar riesgos en el deporte. Así, algunos individuos pueden sobreestimar su capacidad o percibirse a sí mismos más capaces de lo que realmente son (Latorre y Pantoja, 2013). Una sobreestimación de la capacidad puede dar lugar a juicios erróneos (Bandura, 1986) y, por lo tanto, a conductas de mayor riesgo. Mediante un análisis bayesiano para la determinación de las variables relevantes de la lesión deportiva Garcia-Mas, Pujals, Fuster-Parra, Núñez y Rubio (2014), indican que una elevada probabilidad de autoeficacia produce dependencia estadística de más probabilidad de lesión deportiva. Por todo ello, entendemos la CP como el grado de autoconocimiento y de aceptación de las propias cualidades y límites.

4. Percepción del riesgo (PR). Kontos (2004) destaca que la percepción del riesgo, la aceptación de éste y la estimación de la propia capacidad, representan importantes factores de riesgo psicológico para las lesiones deportivas de los adolescentes. Además, los sujetos con altos niveles de toma de riesgos, bajos niveles de riesgo percibido y bajos niveles de estimación de habilidad, presentan un mayor riesgo de lesión (Kontos, 2004). Chamarro, Rovira y Fernández-Castro (2010), en su trabajo centrado en personas excursionistas, indican que en la determinación del nivel de preocupación (como medida de PR), la dificultad y la prisa se combinan interactivamente y que dichas variables, como bloque, interactúan a su vez con la fatiga, y con la confianza. De este modo, el factor PR define el grado de identificación de las situaciones arriesgadas.

5. Competitividad (COMP). La ansiedad competitiva puede ser un factor de predisposición a la lesión (Olmedilla, Andreu, Ortín y Blas, 2009). En este mismo sentido, Maddison y Prapavessis (2005) indican que la elevada ansiedad competitiva, en una situación de estrés, provoca más riesgo de lesión. En relación a este factor, Llopis y Vilanova (2015) afirman que los corredores presentan un mayor grado de competitividad que de interés por la búsqueda de aventura o por la evasión. Así pues, la COMP hace referencia al esfuerzo de una persona para dominar una tarea o alcanzar la excelencia y la motivación enfocada hacia el logro de objetivos en el ámbito deportivo (Prieto, Labisa y Olmedilla, 2014). Así, concebimos la COMP como el factor que induce al atleta en centrarse que es mejor a los demás, a arriesgarse y a perder el objetivo principal de su rendimiento.

\section{MÉTODO}

\section{Participantes}

La muestra utilizada en el presente estudio se compuso de un total de 237 sujetos con edades comprendidas entre 17 y 60 años $(38,37 \pm 8,37)$, de los cuales 183 sujetos fueron hombres $(77,2 \%)$ y 54 mujeres $(22,8 \%)$. Se trata de una muestra no probabilística, por conveniencia, obtenida en 3 carreras distintas. Tal y como se puede apreciar en la siguiente Tabla 1. Características de la muestra., se diferencian 6 
grupos, que pertenecen a las distintas distancias organizadas en cada evento: dos grupos del Cros de Muntanya Can Caralleu (7,5 km y $15 \mathrm{~km})$; tres grupos de la Marató Borredà-Xtrai $(11 \mathrm{~km}, 28 \mathrm{~km}$ y $44 \mathrm{~km})$; y el sexto grupo está conformado por los participantes de la Zurich Marató de Barcelona (42 km).

Tabla 1. Características de la muestra.

\begin{tabular}{|c|c|c|c|c|c|c|c|c|}
\hline \multirow{2}{*}{ Carreras } & \multirow{2}{*}{ Distancias } & \multirow[b]{2}{*}{$\mathrm{n}$} & \multicolumn{2}{|c|}{ Edad } & \multicolumn{2}{|c|}{ Hombres } & \multicolumn{2}{|c|}{ Mujeres } \\
\hline & & & Media & DT & $\mathrm{n}$ & $\%$ & $\mathrm{n}$ & $\%$ \\
\hline \multirow{3}{*}{ Cros de Muntanya Can Caralleu } & $7,5 \mathrm{~km}$ & 24 & 40,17 & 6,86 & 16 & 66,7 & 8 & 33,3 \\
\hline & $15 \mathrm{~km}$ & 72 & 39,5 & 8,21 & 59 & 81,9 & 13 & 18,1 \\
\hline & Total Caralleu & 96 & 39,67 & 7,86 & 75 & 78,1 & 21 & 21,9 \\
\hline \multirow{4}{*}{ Borredà-Xtrail } & $11 \mathrm{~km}$ & 13 & 37,46 & 10,97 & 7 & 53,8 & 6 & 46,2 \\
\hline & $28 \mathrm{~km}$ & 36 & 36,36 & 8,89 & 26 & 72,2 & 10 & 27,8 \\
\hline & $44 \mathrm{~km}$ & 47 & 36,21 & 7,66 & 40 & 85,1 & 7 & 14,9 \\
\hline & Total Borredà & 96 & 36,44 & 8,54 & 73 & 76 & 23 & 24 \\
\hline \multirow[t]{2}{*}{ Zurich Marató de Barcelona } & $42 \mathrm{~km}$ & 45 & 39,7 & 8,44 & 35 & 77,8 & 10 & 22,2 \\
\hline & Total muestra & 237 & 38,37 & 8,37 & 183 & 77,2 & 54 & 22,8 \\
\hline
\end{tabular}

\section{Perfil sociodemográfico e historial de lesiones de la muestra}

En la Tabla 2; Error! No se encuentra el origen de la referencia., se presentan los resultados de los ítems relativos al perfil sociodemográfico de los corredores. Además de los datos sobre el sexo y la edad de los participantes, que se encuentran en la Tabla 1. Características de la muestra., se puede observar que: en referencia a la situación laboral, la mayor parte son empleados por cuenta ajena $(57,4 \%)$, funcionarios $(11,8 \%)$ y autónomos $(9,3 \%)$; los estudios más altos alcanzados son universitarios $(36,7 \%)$, formación profesional $(21,5 \%)$ y postgrado $(19 \%)$; la mayor parte tiene experiencia previa en eventos de larga distancia $(78,5 \%)$; realizan una media de 4 entrenamientos a la semana en los últimos 3 meses; entrenando una media de más de 7 horas a la semana.. Por lo que hace referencia al historial de lesiones, se observa que la media de lesiones en los últimos 3 años es de 1,51 $\pm 1,97$,

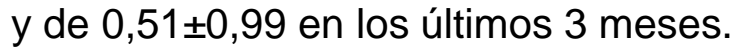


Tabla 2. Descripción sociodemográfica de la muestra

\begin{tabular}{|c|c|c|c|c|c|}
\hline & & $\begin{array}{c}\text { Cros de } \\
\text { Muntanya } \\
\text { Can } \\
\text { Caralleu } \\
(n=96)\end{array}$ & $\begin{array}{c}\text { Borredà- } \\
\text { Xtrail } \\
(n=96)\end{array}$ & $\begin{array}{c}\text { Zurich } \\
\text { Marató de } \\
\text { Barcelona } \\
(n=45)\end{array}$ & $\begin{array}{c}\text { Total de la } \\
\text { muestra } \\
(n=237)\end{array}$ \\
\hline \multirow{8}{*}{ Dedicación laboral n (\%) } & Estudiante & $2(2,1)$ & $10(10,4)$ & $2(4,4)$ & $12(5,9)$ \\
\hline & Parado/a & $1(1,0)$ & $3(3,1)$ & $2(4,4)$ & $6(2,5)$ \\
\hline & Funcionario/a & $11(11,5)$ & $11(11,5)$ & $6(13,3)$ & $28(11,8)$ \\
\hline & Autónomo/a & $9(9,4)$ & $9(9,4)$ & $4(8,9)$ & $22(9,3)$ \\
\hline & Empleado/a & $65(67,7)$ & $50(52,1)$ & $21(46,7)$ & $136(57,4)$ \\
\hline & Empresario/a & $4(4,2)$ & $5(5,2)$ & $6(13,3)$ & $15(6,3)$ \\
\hline & $\begin{array}{l}\text { Deportista } \\
\text { profesional }\end{array}$ & $0(0)$ & $1(1,0)$ & $0(0)$ & $1(0,4)$ \\
\hline & Otros & $4(4,2)$ & $7(7,3)$ & $4(8,9)$ & $15(6,3)$ \\
\hline \multirow{7}{*}{$\begin{array}{l}\text { Estudios más altos } \\
\text { alcanzados } \mathrm{n}(\%)\end{array}$} & $\begin{array}{l}\text { Estudios } \\
\text { primarios }\end{array}$ & $9(9,4)$ & $9(9,4)$ & $4(8,9)$ & $22(9,3)$ \\
\hline & $\begin{array}{l}\text { Formación } \\
\text { profesional }\end{array}$ & $19(19,8)$ & $21(21,9)$ & $11(24,4)$ & $51(21,5)$ \\
\hline & Bachillerato & $13(13,5)$ & $10(10,4)$ & $6(13,3)$ & $29(12,2)$ \\
\hline & $\begin{array}{c}\text { Estudios } \\
\text { universitarios }\end{array}$ & $38(39,6)$ & $33(34,4)$ & $16(35,6)$ & $87(36,7)$ \\
\hline & $\begin{array}{l}\text { Estudios } \\
\text { postgrado }\end{array}$ & $15(15,6)$ & $22(22,9)$ & $8(17,8)$ & $45(19)$ \\
\hline & Doctorado & $2(2,1)$ & $1(1,0)$ & $0(0)$ & $3(1,3)$ \\
\hline & Otros & $0(0)$ & $0(0)$ & $0(0)$ & $0(0)$ \\
\hline \multirow{2}{*}{ Experiencia previa $\mathrm{n}(\%)$} & Sí & $61(63,5)$ & $89(92,7)$ & $36(80)$ & $186(78,5)$ \\
\hline & No & $35(36,5)$ & $7(7,3)$ & $9(20)$ & $51(21,5)$ \\
\hline \multicolumn{2}{|c|}{$\begin{array}{l}\text { Entrenamiento últimos } 3 \text { meses } \\
\text { (sesiones por semana). Media } \pm \mathrm{DT}\end{array}$} & $3,54 \pm 1,53$ & $4,22 \pm 2,11$ & $4,76 \pm 2,17$ & $4,05 \pm 1,96$ \\
\hline \multicolumn{2}{|c|}{$\begin{array}{c}\text { Entrenamiento últimos } 3 \text { meses (horas } \\
\text { por semana). Media } \pm \mathrm{DT}\end{array}$} & $5,56 \pm 3,53$ & $8,60 \pm 7,42$ & $8,53 \pm 5,16$ & $7,35 \pm 5,86$ \\
\hline \multicolumn{2}{|c|}{ Lesiones 3 años, Media \pm DT } & $1,38 \pm 2,30$ & $1,85 \pm 1,89$ & $1,07 \pm 1,10$ & $1,51 \pm 1,97$ \\
\hline \multicolumn{2}{|c|}{ Lesiones 6 meses. Media \pm DT } & $0,51 \pm 1,19$ & $0,61 \pm 0,92$ & $0,27 \pm 0,50$ & $0,51 \pm 0,99$ \\
\hline
\end{tabular}

\section{Instrumento}

La técnica de recopilación de datos utilizada para medir el constructo propensión al accidente deportivo ha sido la escala PAD-22 de Latorre y Pantoja (2013). Es un cuestionario de autoinforme compuesto por 22 ítems que describen unas variables que condicionan la posibilidad de sufrir un accidente deportivo (Latorre y Pantoja, 2013). El sujeto debe responder las cuestiones según su nivel de conformidad con las oraciones, siguiendo una escala tipo Likert, del 1 al 6 , siendo 1 , muy en desacuerdo y 6 , muy de acuerdo.

La escala de Latorre y Pantoja (2013) mostró fiabilidad, con una buena consistencia interna con un coeficiente alfa de Cronbach igual a .82, y una adecuada validez de contenido y factorial. 
Para la adaptación del cuestionario, se complementaron los pasos de elaboración de Latorre y Pantoja (2013). Se revisaron los trabajos relacionados con los factores psicológicos y de personalidad propios del sujeto que condicionan la accidentalidad en el deporte. Posteriormente, se validó el contenido de la adaptación del cuestionario, solicitando un juicio crítico a un grupo de cuatro expertos del campo, con experiencia investigadora en el ámbito de la accidentalidad deportiva.

Finalmente, se mantuvieron los 22 ítems del trabajo de Latorre y Pantoja (2013), basados en las dimensiones mencionadas: Búsqueda de Sensaciones (BS), Asunción de Riesgos (AR), Competencia Percibida (CP), Percepción del Riesgo (PR) y Competitividad (COMP). El factor BS está formado por 6 ítems del cuestionario, mientras que todos los demás están compuestos por 4 ítems cada uno.

Asimismo, se han añadido al cuestionario preguntas para definir el perfil sociodemográfico, los hábitos deportivos y el número de lesiones de los sujetos integrantes de la muestra. Por un lado, se han añadido preguntas relacionadas con las variables de sociotipo: año de nacimiento, género, lugar de residencia habitual, dedicación laboral y estudios alcanzados; por otro lado, las variables para conocer sus hábitos deportivos: experiencia previa en la participación en eventos de larga distancia, frecuencia de entrenamiento -número de sesiones y media de horas semanales de entrenamiento-; y, finalmente, el número de lesiones sufridas en los últimos 3 años y en los últimos 6 meses.

\section{Procedimiento de administración}

Previamente a la administración de los cuestionarios, los sujetos fueron informados de la confidencialidad de sus respuestas, así como del carácter voluntario de su participación, consintiendo el tratamiento de la información aportada con el cumplimiento del cuestionario, siendo garantizado su anonimato en todo momento. El cuestionario fue autoadministrado, con pequeñas diferencias según los eventos analizados: a los sujetos de la Zurich Marató de Barcelona se les administró el cuestionario al finalizar la carrera, por pequeños grupos y en presencia de un investigador o colaborador del estudio; a los sujetos del Cros de Muntanya Can Caralleu, se les administró el cuestionario el día de la recogida de dorsales y el mismo día de la carrera; a los sujetos del Marató Borredà-Xtrail se les administró el cuestionario adaptado en formato online mediante el uso de la herramienta Google Forms (Google Inc. Mountain View, USA)..La duración de administración de los cuestionarios fue de aproximadamente 8 minutos por participante.

\section{Análisis estadístico}

Para el análisis de datos se recorrió al empleo del programa estadístico SPSS., v.18.0 para Windows, (SPSS Inc, Chicago, USA). Se asume el supuesto de normalidad de la muestra, y como consecuencia se realizan pruebas paramétricas. Se ha utilizado la estadística descriptiva (medias, DT), correlaciones (Pearson) y diferencias de medias (ANOVA y Prueba t), empleando como covariable el sexo en la comparación de los grupos de deportistas. El nivel de significación fue de $p<.01$. 


\section{RESULTADOS}

\section{Factores de propensión al accidente deportivo, valor total de propensión $y$ frecuencia de lesiones}

En la Tabla 3. Correlación de Pearson entre factores, propensión al accidente y lesiones., se puede observar una correlación significativa entre los factores y el valor resultante total de propensión al accidente deportivo. A pesar de no haberse podido identificar una correlación significativa entre los factores y el historial de lesiones de los sujetos, sí existe correlación entre en el valor resultante total de propensión al accidente y el número de lesiones sufridas en los últimos 6 meses.

Tabla 3. Correlación de Pearson entre factores, propensión al accidente y lesiones.

\begin{tabular}{ccccccccc}
\hline & BS & AR & CP & PR & COMP & $\begin{array}{c}\text { Total } \\
\text { PAD-22 }\end{array}$ & L3A & L6M \\
\hline BS & 1 &, $333^{* *}$ & &, $527^{* *}$ & &, $795^{* *}$ & & \\
AR & & 1 & &, $363^{* *}$ & &, $584^{* *}$ & & \\
CP & & & 1 &, $295^{* *}$ &, $460^{* *}$ &, $570^{* *}$ & & \\
PR & & & & 1 &, $244^{* *}$ &, $727^{* *}$ & \\
COMP & & & & & 1 &, $602^{* *}$ & & \\
PAD & & & & & & 1 & &, $241^{* *}$ \\
\hline
\end{tabular}

${ }^{\star *}$. $\mathrm{p}<0,01$. Búsqueda de sensaciones (BS). Asunción de riesgos (AR). Competencia percibida (CP). Percepción del riesgo (PR). Competitividad (COMP). Propensión al Accidente Deportivo (PAD-22). Número de lesiones últimos 3 años (L3A). Número de lesiones últimos 6 meses (L6M).

Relación entre factores de propensión al accidente, valor total de propensión, carreras, distancias, sexo y otros factores sociodemográficos

En la Tabla 4. Escala PAD-22 por carreras., se muestran las medias de los factores de propensión y del valor total de propensión de cada carrera, y la comparación por carreras de los valores medios de los factores y total. De forma no significativa, se observa que: los corredores de Cros de Muntanya Can Caralleu obtienen puntuaciones inferiores en BS, CP y PR; los corredores de la Borredà-Xtrail tienden a obtener valores más elevados en BS, AR y PR, en comparación con los demás grupos; y los sujetos de la Zurich Marató de Barcelona presentan puntuaciones más elevadas en CP, COMP y el valor total de PAD-22, y valores inferiores a AR, en comparación con los participantes en las otras carreras. Se observan diferencias significativas $(p<.05)$ en las dimensiones de CP y COMP. En las comparaciones por pares mediante ajuste Bonferroni, se encuentran diferencias significativas $(p<.05)$ entre los participantes en la Zurich Marató de Barcelona y los de Borredà-Xtrail en la dimensión de COMP, mientras que los corredores de la Marató de Barcelona presentan una mayor puntuación. En la dimensión CP, los participantes de la Marató de Barcelona en relación con los corredores del Cros de Muntanya Can Caralleu obtienen mayores valores de COMP $(p<.05)$; tal y como ocurre en la Borredà-Xtrail con respecto a la Marató de Barcelona $(p<.05)$. 
Tabla 4. Escala PAD-22 por carreras.

\begin{tabular}{cccccccc}
\hline & $\begin{array}{c}\text { Cros de Muntanya Can } \\
\text { Caralleu } \\
(n=96)\end{array}$ & \multicolumn{2}{c}{$\begin{array}{c}\text { Borredà-Xtrail } \\
(n=96)\end{array}$} & \multicolumn{2}{c}{$\begin{array}{c}\text { Zurich Marató de } \\
\text { Barcelona } \\
(n=45)\end{array}$} & \\
& $\mathrm{M}$ & $\mathrm{DT}$ & $\mathrm{M}$ & $\mathrm{DT}$ & $\mathrm{M}$ & $\mathrm{DT}$ & $\mathrm{F}$ \\
\cline { 2 - 7 } $\mathrm{BS}$ & 17,23 & 6,71 & 19,19 & 7,07 & 17,38 & 6,67 & 2,23 \\
AR & 6,8 & 3,23 & 7,13 & 3,61 & 6,38 & 3,56 & 0,73 \\
CP & 9,23 & 2,93 & 9,45 & 2,58 & 10,8 & 3,96 & $4,39^{*}$ \\
PR & 8,47 & 3,68 & 8,82 & 3,43 & 8,67 & 4,68 & 0,21 \\
COMP & 9,83 & 4,21 & 9,04 & 3,91 & 11,33 & 5,11 & $4,40^{*}$ \\
Total PAD- & 51,56 & 13,81 & 53,63 & 14,06 & 54,56 & 17,24 & 0,81 \\
22 & & & & & & &
\end{tabular}

Datos expresados en media (M) y desviación típica (DT). Búsqueda de sensaciones (BS). Asunción de riesgos (AR). Competencia percibida (CP). Percepción del riesgo (PR). Competitividad (COMP). Propensión al Accidente Deportivo (Total PAD-22). ${ }^{*} p<.05$.

En la Tabla 5. Escala PAD-22 por distancias.se muestran de forma detallada los valores medios de los factores de propensión al accidente deportivo y el total de los factores por distancias, y la comparación entre ellas. De forma no significativa, se observa que: a medida que aumenta la distancia de la carrera de la Borredà-Xtrail $(11 \mathrm{~km}, 28 \mathrm{~km}$ y $44 \mathrm{~km})$, aumenta la BS, mientras que el resto de distancias tienen valores inferiores a éstas. En relación al factor AR, son los corredores de las distancias superiores a los $40 \mathrm{~km}$ los que obtiene junto con la distancia $7,5 \mathrm{~km}$, puntuaciones inferiores al resto. En lo que a PR se refiere, se muestra una tendencia a obtener puntuaciones más elevadas a medida que aumenta la distancia recorrida, con la excepción de los participantes de los $28 \mathrm{~km}$, que tienen valores superiores al resto. Para los valores totales de PAD-22, se observa que a medida que aumentan las distancias, tienden a aumentar estos valores, con las excepciones de los participantes en los $11 \mathrm{~km}$ y los $42 \mathrm{~km}$. Se observan diferencias significativas $(p<.05)$ en las dimensiones de CP y COMP. Los corredores tienden a obtener mayores puntuaciones en CP, a medida que aumenta la distancia de la carrera en la que participan, con la excepción de los participantes en la distancia de $44 \mathrm{~km}$, que obtienen valores inferiores a los de $28 \mathrm{~km}$ y $42 \mathrm{~km}$. Por lo que hace referencia al factor COMP, los sujetos que obtienen valores inferiores, por orden ascendente, son los de $28 \mathrm{~km}, 7,5 \mathrm{~km}$ y $44 \mathrm{~km}$; en el resto de casos, obtienen mayores puntuaciones a medida que aumenta la distancia.

Tabla 5. Escala PAD-22 por distancias.

\begin{tabular}{|c|c|c|c|c|c|c|c|c|c|c|c|c|c|}
\hline & \multicolumn{2}{|c|}{$\begin{array}{l}7,5 \mathrm{~km} \\
(\mathrm{n}=24)\end{array}$} & \multicolumn{2}{|c|}{$\begin{array}{l}11 \mathrm{~km} \\
(\mathrm{n}=13)\end{array}$} & \multicolumn{2}{|c|}{$\begin{array}{l}15 \mathrm{~km} \\
(\mathrm{n}=72)\end{array}$} & \multicolumn{2}{|c|}{$\begin{array}{l}28 \mathrm{~km} \\
(\mathrm{n}=36)\end{array}$} & \multicolumn{2}{|c|}{$\begin{array}{l}42 \mathrm{~km} \\
(\mathrm{n}=45)\end{array}$} & \multicolumn{2}{|c|}{$\begin{array}{l}44 \mathrm{~km} \\
(\mathrm{n}=47)\end{array}$} & \multirow[b]{2}{*}{$F$} \\
\hline & M & DT & $M$ & DT & $M$ & DT & $M$ & DT & M & DT & M & DT & \\
\hline BS & 17,33 & 7,94 & 18,15 & 6,56 & 17,19 & 6,31 & 18,58 & 7,42 & 17,38 & 6,67 & 19,94 & 7,01 & 1,11 \\
\hline AR & 6,21 & 2,7 & 7,46 & 4,01 & 7 & 3,39 & 7,78 & 4,21 & 6,38 & 3,56 & 6,53 & 2,93 & 1,05 \\
\hline $\mathrm{CP}$ & 8,33 & 2,9 & 9,38 & 2,53 & 9,53 & 2,9 & 10,03 & 3,11 & 10,8 & 3,96 & 9,02 & 2,06 & $2,80^{*}$ \\
\hline PR & 7,79 & 3,79 & 8,15 & 3,24 & 8,69 & 3,64 & 9,14 & 3,38 & 8,67 & 4,68 & 8,77 & 3,56 & 0,42 \\
\hline COMP & 8,75 & 4,16 & 10,08 & 5,04 & 10,19 & 4,19 & 8,72 & 3,46 & 11,33 & 5,11 & 9 & 3,93 & $2,36^{*}$ \\
\hline $\begin{array}{c}\text { Total } \\
\text { PAD-22 }\end{array}$ & 48,42 & 16,49 & 53,23 & 16,51 & 52,61 & 12,76 & 54,25 & 14,32 & 54,56 & 17,24 & 53,26 & 13,45 & 0,64 \\
\hline
\end{tabular}


Datos expresados en media (M) y desviación típica (DT). Búsqueda de sensaciones (BS). Asunción de riesgos (AR). Competencia percibida (CP). Percepción del riesgo (PR). Competitividad (COMP). Propensión al Accidente Deportivo (Total PAD-22). ${ }^{*} \mathrm{p}<.05$.

En la Tabla 6. Escala PAD-22 según el sexo.se exponen los valores medios de los factores PAD-22 según el sexo, haciendo además la comparación entre ambos. Los varones, por lo general, muestran mayor puntuación que las mujeres, con diferencias significativas con $\mathrm{p}<.005$, en las dimensiones BS y en el valor resultante total de propensión al accidente y con $\mathrm{p}<.01$ en la dimensión PR. De forma no significativa, se muestra una tendencia de los hombres a tener valores superiores en AR y COMP que las mujeres, por otro lado, son las mujeres las que obtienen mayores puntuaciones en $\mathrm{CP}$.

Tabla 6. Escala PAD-22 según el sexo.

\begin{tabular}{cccccc}
\hline & \multicolumn{2}{c}{$\begin{array}{c}\text { Hombre } \\
(\mathrm{n}=183)\end{array}$} & \multicolumn{2}{c}{$\begin{array}{c}\text { Mujer } \\
(\mathrm{n}=54)\end{array}$} & \\
\cline { 2 - 5 } & $\mathrm{M}$ & $\mathrm{DT}$ & $\mathrm{M}$ & $\mathrm{DT}$ & $\mathrm{F}$ \\
\hline BS & 18,87 & 6,69 & 15,28 & 6,89 & $11,85^{* *}$ \\
AR & 7,06 & 3,49 & 6,15 & 3,24 & 2,94 \\
CP & 9,55 & 3,01 & 9,83 & 3,27 & 0,35 \\
PR & 9,01 & 3,79 & 7,43 & 3,49 & $7,54^{*}$ \\
COMP & 9,97 & 4,35 & 9,2 & 4,29 & 1,31 \\
Total PAD-22 & 54,46 & 14,39 & 47,89 & 14,3 & $8,73^{* *}$ \\
\hline
\end{tabular}

Datos expresados en media y desviación típica (DT). Búsqueda de sensaciones (BS). Asunción de riesgos (AR). Competencia percibida (CP). Percepción del riesgo (PR). Competitividad (COMP). Propensión al Accidente Deportivo (Total PAD-22). * $p<.01 .{ }^{*} p<.005$

En relación al resto de variables sociodemográficas analizadas, no se han encontrado diferencias significativas entre factores, tampoco existen diferencias significativas de los ítems sociodemográficos y del historial de lesiones en las diferentes carreras o distancias.

\section{DISCUSIÓN}

Mediante el presente estudio se han tratado de identificar a los corredores por montaña que presentan mayor propensión a la lesión deportiva, analizando la influencia del tipo de carrera y la distancia en la que participan, así como el sexo y otros factores sociodemográficos.

El análisis descriptivo de la muestra permite realizar una aproximación al perfil sociodemográfico y deportivo de los corredores. Por lo general, se trata de varones, de entre 30 y 46 años, asalariados, con estudios universitarios, formación profesional y/o postgrado, con experiencia previa en eventos de larga distancia, entrenan una media de 4 veces y un total de 7 horas a la semana. Estos datos coinciden de forma general con los estudios realizados sobre el perfil de los participantes en carreras populares (Llopis y Llopis, 2012; Llopis y Vilanova, 2015), también en el estudio del perfil de los participantes en raids de aventura (Baena, 2008), y siendo, todavía más próximo al estudio del perfil de los corredores de ultramaratones de Hoffman y Fogard (2012). 
Los resultados obtenidos mediante el uso de la escala PAD-22, en función de la carreras, sugieren que los corredores de la maratón sobre asfalto difieren de los corredores por montaña en cuanto a CP y COMP; los corredores de maratón sobre asfalto han obtenidos mayores puntuaciones en CP y, por lo tanto, serían más propensos a realizar conductas arriesgadas, correspondiendo así con Bandura (1997). Por otro lado, también son los corredores de maratón sobre asfalto los que tienen mayor grado de COMP y, por lo tanto, una mayor propensión al accidente deportivo. Los resultados obtenidos coinciden con los expuestos por Llopis y Vilanova (2015), reafirmando que los corredores de maratones dan más importancia al hecho de competir que a la búsqueda de nuevas sensaciones, en comparación con los corredores por montaña.

En comparación con el estudio hecho sobre jugadores de rugby, voleibol y deporte recreativo de Latorre y Pantoja (2013), las puntuaciones en las distintas dimensiones han sido en todos los casos inferiores, excepto en un único caso: el factor $\mathrm{CP}$, en el que los participantes en la maratón sobre asfalto obtienen una puntuación más elevada que los practicantes de Voleibol. Este dato sugiere que los corredores por montaña y los participantes en la maratón son menos propensos al accidente deportivo que los jugadores de rugby, voleibol y deporte recreativo. En esta línea, según la recopilación de Gomà-i-Freixanet et al. (2012), se correspondería que los corredores de montaña y de asfalto son considerados deportistas de bajo riesgo, en comparación con los alpinistas, considerados de riesgo elevado (Jack y Ronan, 1998) y con los participantes en la Ultramaratón de Idiasport, consideradores de riesgo intermedio (Hughes et al., 2003).

En relación a la consideración de otras variables sociodemográficas, no se han encontrado diferencias significativas que evidencien una relación comparativa entre los distintos factores analizados, aunque si que se han encontrado cierto tipo de correlación en función del sexo, donde los valores en COMP de los corredores hombres son más elevados que los de las mujeres, confirmando los resultados obtenidos en estudios relacionados previos (Acevedo, Dzewaltowski, Gill y Noble, 1992; Gill, Williams, Dowd, Beaudoin y Martin, 1996). También es así en el estudio sobre distintos deportes de Latorre y Pantoja (2013); en éste, además, se sugiere una diferencia en la BS, mayor en hombres que en mujeres. Nuestro estudio no muestra diferencias significativas entre hombres y mujeres en COMP, pero sí lo hace en la dimensión BS, en la que los hombres muestran valores más elevados que las mujeres, del mismo modo que en el Total PAD-22. Esto nos permite corroborar, en la línea de estudios previos (Byrnes, Miller y Schafer, 1999), que los hombres son más propensos al accidente deportivo que las mujeres.

\section{CONCLUSIÓN}

El estudio presentado nos ha permitido analizar la propensión al accidente deportivo en función del tipo de carreras y distancias participadas, pudiendo comparar, también, los corredores de una maratón sobre asfalto con los corredores de una maratón por montaña. Hemos podido constatar como los corredores de la maratón por asfalto tienen una sobreestimación de la CP y grados de COMP mayores a los de los corredores de montaña; y, por otro lado, los hombres persiguen más la BS y son más propensos al accidente deportivo que las mujeres. 
No se ha encontrado una significativa relación entre los resultados de las dimensiones obtenidas en la escala PAD-22 (Latorre y Pantoja, 2013) y el historial de lesiones de los deportistas, pero sí una relación entre el total de la escala PAD22 y el número de lesiones en los últimos 6 meses; de este modo, se considera que, aunque cabría aumentar la muestra para afirmarlo con mayor fiabilidad, la escala podría ofrecernos una previsión aproximada de la propensión al accidente deportivo.

El estudio sugiere que los participantes de la maratón por asfalto tienden a tener más propensión al accidente que los corredores por montaña, experimentando diferencias significativas en la CP y COMP, como dimensiones con mayor puntuación para los corredores en la maratón de asfalto. Además, podemos concluir que los participantes, tanto de trail running como de running por asfalto, tienden a una baja propensión al accidente, puesto que, tanto los corredores por montaña, independientemente de la distancia que recorran, como los maratonianos por asfalto obtienen valores menores en las diferentes dimensiones de propensión al accidente que los de la muestra de practicantes de rugby, voleibol o deportes recreativos del estudio de Latorre Román y Pantoja Vallejo (2013).

Por otro lado, el estudio nos sugiere que los hombres participan más en este tipo de eventos por razones de BS y, por lo general, son más propensos al accidente deportivo.

En relación a las limitaciones del presente estudio que generan futuras líneas de trabajo, se considera que sería necesario, tal y como apuntan Latorre y Pantoja, 2013, realizar un seguimiento preciso de la accidentabilidad y las lesiones de los deportistas, para poder comprobar mayores concordancias con la escala PAD-22. Por otro lado, sería interesante poder ampliar estos resultados a una mayor muestra, para hacer plausible su representatividad, así como ampliar el estudio a corredores de distancias más largas, teniendo en cuenta el gran auge de las carreras de ultradistancia en montaña.

\section{REFERENCIAS BIBLIOGRÁFICAS}

Acevedo, E. O., Dzewaltowski, D. A., Gill, D. L., \& Noble, J. M. (1992). Cognitive orientations of ultramarathoners. Sport Psychologist, 6(3), 242-253. https://doi.org/10.1123/tsp.6.3.242

Andersen, M. B., \& Williams, J. M. (1988). A Model of Stress and Athletic Injury: Prediction and Prevention. Journal of Sport \& Exercise Psychology, 10, 294306. https://doi.org/10.1123/jsep.10.3.294

Baena, A. (2008). Análisi del perfil sociodemográfico y deportivo de los competidores de raids de aventura en España. Granada. Retrieved from http://0-hera.ugr.es.adrastea.ugr.es/tesisugr/17367359.pdf

Bahr, R., \& Krosshaug, T. (2005). Understanding injury mechanisms: a key component of preventing injuries in sport. British Journal of Sports Medicine, 39(6), 324-329. https://doi.org/10.1136/bjsm.2005.018341

Bandura, A. (1986). Social foundations of thought and action: A social cognitive theory. Englewood Cliffs: Patience-Hall, Inc.

Bandura, A. (1997). Self-efficacy: The exercise of control. New York.

Byrnes, J. P., Miller, D. C., \& Schafer, W. D. (1999). Gender differences in risk taking: 
A meta-analysis. Psychological Bulletin, (125), 367-383. https://doi.org/10.1037/0033-2909.125.3.367

Chamarro, A., Rovira, T., \& Fernández-Castro, J. (2010). Juicios de riesgo en el deporte: una aproximación experimental en excursionistas. Revista de Psicologia Del Deporte, 19(2), 203-217.

Clawson, M., \& Knetsch, J. L. (2013). Economics of outdoor recreation (Vol. 3). New York/London: RFF Press. https://doi.org/10.4324/9781315064215

De la Vega, R., Rivera, O., \& Ruiz, R. (2011). Personalidad resistente en carreras de fondo: Comparativa entre ultra fondo y diez kilómetros. Revista de Psicologia Del Deporte, 20(2), 445-454.

Dienot, J., \& Theiller, D. (1999). Les Nouveaux Loisirs Sportifs en Montagne. Les Aventuriers du Quotidien. Publications de La Maison Des Sciences de l'homme d'Aquitaine.

Garcia-Mas, A., Pujals, C., Fuster-Parra, P., Núñez, A., \& Rubio, V. J. (2014). Determinación de las variables psicológicas y deportivas relevantes a las lesiones deportivas: Un análisis bayesiano. Revista de Psicologia Del Deporte, 23(2), 423-429.

García Ferrando, M., \& Llopis, R. (2011). Ideal democrático y bienestar personal. Encuesta sobre los hábitos deportivos en España 2010. Madrid: Centro de Investigaciones Sociológicas. Retrieved from http://www.csd.gob.es/csd/estaticos/dep-soc/encuesta-habitosdeportivos2010.pdf

Generalitat de Catalunya. (2015). Comparativa salvaments 2010-14. Retrieved June 23, 2015 , from http://interior.gencat.cat/ca/arees_dactuacio/bombers/seguretat_a_la_muntan ya/salvaments_al_medi_natural/

Gill, D. L., Williams, L., Dowd, D. A., Beaudoin, C. M., \& Martin, J. J. (1996). Competitive orientations and motives of adult sport and exercise participants. Journal of Sport Behavior, 19(4), 307-318.

Gomà-i-Freixanet, M., Martha, C., \& Muro, A. (2012). Does the Sensation-Seeking trait differ among participants engaged in sports with different levels of physical risk? Anales de Psicología, 28(1), 223-232.

González, A. X., \& Mundina, J. J. (2014). Experiencia práctica: Actividades físicas y deportivas en el medio natural. Enseñando mediante proyectos en el ámbito universitario. Revista Española de Educación Física y Deportes: REEFD, 405, 97-104. Retrieved from http://reefd.es/index.php/reefd/article/view/40/42

Hanson, S. (1992). The Relationship of Personality Characteristics, Life Stress, and Coping Resources to Athletic Injury. Journal of Sport and Exercise Psychology, 1(1978), 262-272.

Hashimoto, M., Hagura, N., Kuriyama, T., \& Nishiyamai, M. (2006). Motivations and psychological characteristics of Japanese ultra-marathon runners using MyersBriggs type indicator. Japanese Journal of Health and Human Ecology, 72(1), 15-24. https://doi.org/10.3861/jshhe.72.15

Heil, J. (1993). Psychology of Sport Injury. Champaign, IL: Human Kinetics.

Hoffman, M. D., \& Fogard, K. (2012). Demographic characteristics of 161-km ultramarathon runners. Research in Sports Medicine (Print), 20(1), 59-69. https://doi.org/10.1080/15438627.2012.634707

Horvath, P., \& Zuckerman, M. (1993). Sensation seeking, risk appraisal, and risky 
behavior. Personality and Individual Differences, 14(1), 41-52. https://doi.org/10.1016/0191-8869(93)90173-Z

Hughes, S., Case, H. S., Stuempfle, K., \& Evans, D. (2003). Personality Profiles of Iditasport Ultra-Marathon Participants. Journal of Applied Sport Psychology, 15(3), 256-261. https://doi.org/10.1080/10413200305385

Inglés, E. (2013). Estratègies de gestió de la pràctica esportiva al medi natural per al desenvolupament sostenible: Un estudi de casos sobre governança $i$ "stakeholders." Universitat de Barcelona. Retrieved from http://www.tdx.cat/handle/10803/279214

Jack, S. ., \& Ronan, K. . (1998). Sensation seeking among high- and low-risk sports participants. Personality and Individual Differences, 25(6), 1063-1083. https://doi.org/10.1016/S0191-8869(98)00081-6

Kontos, A. P. (2004). Perceived Risk, Risk Taking, Estimation of Ability and Injury Among Adolescent Sport Participants. Journal of Pediatric Psychology, 29(6), 447-455. https://doi.org/10.1093/jpepsy/jsh048

Latorre, P. Á., \& Pantoja, A. (2013). Diseño y validación de un cuestionario de propensión al accidente deportivo (PAD-22). Cuadernos de Psicología Del Deporte, 13, 51-61. https://doi.org/10.4321/S1578-84232013000100006

Llopis, R., \& Llopis, D. (2012). Una tipologia sociocultural dels corredors populars a Espanya. Apunts Educació Física i Esports, (108), 9-16. https://doi.org/10.5672/apunts.2014-0983.cat.(2012/2).108.01

Llopis, R., \& Vilanova, A. (2015). A Sociological Analysis of the Evolution and Characteristics of Running. In J. Scheerder \& K. Breedveld (Eds.), Running Across Europe: The Rise and Size of One of the Largest Sport Markets (pp. 220-240). Basingstoke: Palgrave Macmillan.

Maddison, R., \& Prapavessis, H. (2005). A Psychological Approach to the Prediction and Prevention of Athletic Injury. Journal of Sport and Exercise Psychology, 27(3), 289-310. https://doi.org/10.1123/jsep.27.3.289

Martos, P. (2002). El Medio Natural como Punto de Encuentro de Turismo y Deporte. Crecimiento y Diversificación. (S. Rebollo \& M. D. Simoes, Eds.). Granada: Universidad de Granada.

Meeuwisse, W. H., Tyreman, H., Hagel, B., \& Emery, C. (2007). A Dynamic Model of Etiology in Sport Injury: The Recursive Nature of Risk and Causation. Clinical Journal of Sport Medicine, 17(3), 215-219. https://doi.org/10.1097/JSM.0b013e3180592a48

Olmedilla, A., Andreu, M. D., Ortín, F. J., \& Blas, A. (2009). Ansiedad competitiva, percepción de éxito y lesiones: un estudio en futbolistas. Revista Internacional de Medicina y Ciencias de La Actividad Física y El Deporte, 9, 51-66.

Osborn, Z. H., Blanton, P. D., \& Schwebel, D. C. (2009). Personality and Injury Risk Among Professional Hockey Players. Journal of Injury and Violence Research, 1(1), 15-19. https://doi.org/10.5249/jivr.v1i1.8

Palmi, J. (2001). Visión psico-social en la intervención de la lesión deportiva. Cuadernos de Psicología Del Deporte, 1, 69-79.

Palmi, J. (2014). Psicología y lesión deportiva: Una breve introducción. Revista de Psicología Del Deporte, 23(2), 389-393.

Prieto Andreu, J. M., Labisa Palmeira, A., \& Olmedilla Zafra, A. (2014). Lesiones deportivas y personalidad: una revisión sistemática. Apunts. Medicina de l'Esport, 49(184), 139-149. https://doi.org/10.1016/j.apunts.2014.06.002 
Ropits Social Running. (2016). Runedia. Retrieved April 29, 2016, from http://www.runedia.com/

Wiese-Bjornstal, D. M., Smith, A. M., Shaffer, S. M., \& Morrey, M. A. (1998). An integrated model of response to sport injury: Psychological and sociological dynamics. Journal of Applied Swrt Psychology, 10(September 2013), 46-69. https://doi.org/10.1080/10413209808406377

Zuckerman, M. (1979). Sensation Seeking: Beyond the Optimal Level of Arousal. Hillsdale, NJ: Erlbaum.

Zuckerman, M. (2009). Sensation seeking. In M. Leary \& R. Hoyle (Eds.), Handbook of Individual Differences in Social behavior (p. 455-465.). New York/London: The Guildford Press.

Zuckerman, M., Eysenck, S., \& Eysenck, H. J. (1978). Sensation seeking in England and America: cross-cultural, age, and sex comparisons. Journal of Consulting and Clinical Psychology, 46(1), 139-149. https://doi.org/10.1037/0022$\underline{006 X .46 .1 .139}$

Número de citas totales / Total references: 42 (100\%)

Número de citas propias de la revista / Journal's own references: $1(2 \%)$

Rev.int.med.cienc.act.fís.deporte - vol. 18 - número 72 - ISSN: 1577-0354 